\title{
Comparison of methods for AmpC $\beta$-lactamase detection in Enterobacteriaceae
}

\author{
Correspondence \\ Tim J. J. Inglis \\ tim.inglis@health.wa.gov.au
}

Received 16 December 2010

Accepted 24 February 2011

\author{
Paul R. Ingram, Tim J. J. Inglis, Tessa R. Vanzetti, Barbara A. Henderson, \\ Gerald B. Harnett and Ronan J. Murray
} Department of Microbiology, PathWest Laboratory Medicine, OEII Medical Centre, Nedlands,
WA 6009, Australia

\begin{abstract}
AmpC $\beta$-lactamases $\left(\mathrm{Bla}_{\mathrm{AmpC}}\right.$ ) are an emerging group of antimicrobial resistance determinants. The lack of an agreed $B \mathrm{~B}_{\mathrm{AmpC}}$ detection method hinders investigation of their epidemiology and understanding of their clinical significance. This study compared the sensitivity and specificity of phenotypic methods of Bla $\mathrm{AmpC}_{\mathrm{Am}}$ detection in a collection of 246 Enterobacteriaceae with a diverse range of $\beta$-lactam resistance profiles. The $\mathrm{Bla}_{\mathrm{AmpC}}$ screening methods evaluated were based on cephamycin, ceftazidime and cefepime susceptibility. These were compared with Bla $\mathrm{AmpC}_{\mathrm{mp}}$ screening using conventional ESBL detection methods. The confirmatory methods evaluated were biologically based assays, inhibitor-based assays, an AmpC Etest and a rapid chromogenic assay. A multiplex nucleic acid amplification test and the three-dimensional enzyme extraction assay were used as reference methods. Bla $\mathrm{AmpC}_{\mathrm{m}}$ activity was present in 74 isolates. The majority of the enzymes were plasmid-encoded and belonged to the CMY, DHA and EBC families. The screening methods had sensitivities between 47 and $99 \%$ and specificities of 45-95\%. The performance of confirmatory tests varied widely, ranging in sensitivity from $19 \%$ to $97 \%$ and in specificity from $88 \%$ to $100 \%$. Only the Tris-EDTA and MAST ID D68C disc tests had a sensitivity and a specificity above $90 \%$. Further investigation is needed to establish the most suitable enzyme substrates, inhibitor types, inhibitor concentrations and interpretative cut-offs in order to refine the inhibitor-based methods.

A simple disc-based protocol using cefoxitin non-susceptibility as a screening tool, followed by

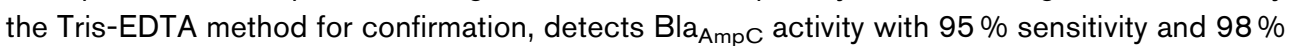
specificity.
\end{abstract}

\section{INTRODUCTION}

In spite of the recent decision by both the Clinical and Laboratory Standards Institute (CLSI) and the European Committee on Antimicrobial Susceptibility Testing (EUCAST) to lower expanded-spectrum cephalosporin breakpoints for Enterobacteriaceae, there remains a need to detect and characterize specific resistance determinants such as extended-spectrum $\beta$-lactamases (ESBLs) and AmpC $\beta$-lactamases ( Bla $_{\mathrm{AmpC}}$ ) for epidemiological surveillance and infection control purposes (Doi \& Paterson, 2007). Although less prevalent than ESBLs (Jacoby, 2009), $\mathrm{Bla}_{\mathrm{AmpC}}$ have a comparatively broader substrate range, are not inhibited by traditional $\beta$-lactamase inhibitors and bacteria producing them are more likely to become resistant to carbapenems (Philippon et al., 2002). Whereas standardized screening and confirmatory methods for ESBL identification are agreed upon (CLSI, 2010), no such methods for Bla $\mathrm{AmpC}_{\mathrm{Amp}}$ detection exist.

Abbreviations: Bla $\mathrm{AmpC}_{\mathrm{A}}$ AmpC $\beta$-lactamase(s); ESBL, extended-spectrum $\beta$-lactamase.
Members of the family Enterobacteriaceae such as Enterobacter spp., Citrobacter freundii and Serratia marcescens possess chromosomally encoded $\mathrm{Bla}_{\mathrm{AmpC}}$, obviating the need for specific $\mathrm{Bla}_{\mathrm{AmpC}}$ testing for these species (Thomson, 2010). Chromosomally encoded Bla $\mathrm{AmpC}_{\mathrm{C}}$ in Escherichia coli is accompanied by negligible expression because of the presence of a transcriptional attenuator coupled with a weak promoter (Tan et al., 2009). Infrequently, hyperproduction of $\mathrm{Bla}_{\mathrm{AmpC}}$ in $E$. coli arises following mutations in these regulatory regions or acquisition of imported $\mathrm{Bla}_{\mathrm{AmpC}}$ genes (Mulvey et al., 2005). Most of the remaining clinically important Enterobacteriaceae, including Klebsiella spp., Proteus mirabilis and Salmonella spp., lack chromosomally encoded $\mathrm{Bla}_{\mathrm{AmpC}}$ but the emergence of plasmid-encoded Bla $_{A m p C}$ in the last few decades has altered the situation. The mobility afforded by transferable genetic elements has enabled rapid global dissemination reminiscent of the emergence of ESBLs (Kohner et al., 2009). For example, in China the prevalence of E. coli and Klebsiella pneumoniae expressing plasmid-encoded Bla $\mathrm{AmpC}_{\mathrm{Ancreased}}$ from $2 \%$ to $9 \%$ between 2005 and 2006 (Ding et al., 2008). 
A number of detection methods for $\mathrm{Bla}_{\mathrm{AmpC}}$ have been proposed. Potential screening tools include reduced susceptibility to cephamycins and/or ceftazidime (Moland et al., 2002), cefepime susceptibility (Doi \& Paterson, 2007) and a positive ESBL screening test (Munier et al., 2010) with or without a negative confirmatory test (Doi \& Paterson, 2007), or combinations of these. More specific, but more time- and labour-intensive tests may be used to confirm the presence of $\mathrm{Bla}_{\mathrm{AmpC}}$, including biological assays [e.g. the modified cefoxitin Hodge test (Yong et al., 2002) and Tris-EDTA disc test (Black et al., 2005a)], inhibitor-based assays [e.g. using boronic acid compounds (Tan et al., 2009) or cloxacillin (Brenwald et al., 2005)], and rapid chromogenic assays (Livermore et al., 2007). Some methods have entered commercial production [e.g. the AmpC Etest (Jacoby, 2009) and MAST ID D68C disc test (Ellem et al., 2009a)], whereas others are less readily available [e.g. Syn 2190 (Doi \& Paterson, 2007), Ro-481220 (Doi \& Paterson, 2007) or LN 2-128 (Black et al., 2005b) inhibitor-based assays]. The comparative sensitivity and specificity of these methods has not been comprehensively studied.

This study aimed to compare the test performance of phenotypic methods for $\mathrm{Bla}_{\mathrm{AmpC}}$ detection in Enterobacteriaceae isolates from a large clinical microbiology laboratory.

\section{METHODS}

Two hundred and forty-six non-duplicate isolates, comprising $165 \mathrm{~K}$. pneumoniae, 63 E. coli, 11 P. mirabilis, 6 Salmonella spp. and one Raoultella sp. were evaluated. The identification of all isolates was confirmed by API 20E and/or Vitek 2 (bioMérieux, France). Ninetysix per cent were clinical isolates, collected between 2006 and 2009 at PathWest Laboratories, QEII Medical Centre, Western Australia. Isolates were cultured from urine $(76 \%)$, blood $(8 \%)$, wound swabs $(6 \%)$, sputum $(4 \%)$, other sterile sites $(4 \%)$ or faeces $(3 \%)$. The isolates were selected to represent a diverse range of $\beta$-lactam resistance profiles, including cefoxitin resistant (77), cefoxitin susceptible (51), ESBL screening test positive/confirmatory test negative (87), confirmed ESBL (23), metallo- $\beta$-lactamase (MBL) carbapenemase (7) and $K$. pneumoniae carbapenemase (KPC) production (1: K. pneumoniae BAA 1705). These isolates had been characterized using either standard CLSI (CLSI, 2010) or published molecular detection methods (Senda et al., 1996). Isolates were stored at $-70{ }^{\circ} \mathrm{C}$ in brain-heart infusion broth with $15 \%$ gycerol then subcultured twice onto Columbia agar base supplemented with $6 \%$ horse blood prior to testing. All tests were performed on MuellerHinton $(\mathrm{MH})$ agar plates of $4 \mathrm{~mm}$ depth. All discs and reagents were stored at $2-8{ }^{\circ} \mathrm{C}$.

The phenotypic tests that were performed once each on all 246 isolates were as follows.

Cephalosporin/aztreonam susceptibility. MICs (cefoxitin, ceftriaxone, cefotaxime, ceftazidime and aztreonam) and/or inhibitory zone sizes (cefoxitin, cefotetan, cefpodoxime) were determined by agar dilution and disc diffusion respectively, as per CLSI recommendations (CLSI, 2009). MICs could not be obtained for P. mirabilis isolates due to the effects of swarming. Susceptibility results were interpreted according to current CLSI criteria (CLSI, 2010).
ESBL screening and confirmatory testing. As per CLSI criteria (CLSI, 2010), isolates with either MIC of $\geqslant 2 \mu \mathrm{g} \mathrm{ml}^{-1}$ (ceftazidime, cefotaxime, ceftriaxone, or aztreonam) or cefpodoxime inhibitory zone diameter $\leqslant 17 \mathrm{~mm}$ (or $\leqslant 22 \mathrm{~mm}$ for $P$. mirabilis) were considered to have a positive ESBL screen. Confirmatory testing was performed using the CLSI disc potentiation method (CLSI, 2010).

Carbapenemase testing. Isolates were screened for carbapenemase production by disc diffusion using a $10 \mu \mathrm{g}$ meropenem disc. In accordance with CLSI recommendations, isolates with an inhibitory zone $\leqslant 21 \mathrm{~mm}$ had carbapenemase production confirmed using the modified (meropenem) Hodge test (CLSI, 2010).

Modified (cefoxitin) Hodge test. This was performed as described by Yong et al. (2002).

Tris-EDTA test. This test, also known as the 'AmpC disc test', was performed as described by Black et al. (2005a) (Fig. 1a).

Saline disc test. This was performed as described by Singhal et al. (2005).

Inhibitor-based tests. Commercially available $6 \mathrm{~mm}$ antibiotic discs were supplemented with either boronic acid compounds or cloxacillin, both known to inhibit $\mathrm{Bla}_{\mathrm{AmpC}}$ activity. A 0.5 McFarland suspension of the test isolate was inoculated evenly on $\mathrm{MH}$ agar, and both unsupplemented and supplemented discs were applied. After incubation for $16-18 \mathrm{~h}$ in room air at $35{ }^{\circ} \mathrm{C}$ the increase in zone size around the supplemented disc compared to the unsupplemented disc was recorded. The antibiotic substrate, inhibitor type, inhibitor concentration and proposed interpretative cut-offs vary among published methods. For the purposes of this study, all zone sizes were reviewed to establish alternative cut-offs that maximized both sensitivity and specificity for Bla $\mathrm{AmpC}_{\mathrm{C}}$ detection.

The combinations of substrate and inhibitor used were phenylboronic acid $(400 \mu \mathrm{g})$ with either cefoxitin $(30 \mu \mathrm{g})$ (Tan et al., 2009) or cefotetan $(30 \mu \mathrm{g})$ (Coudron, 2005), phenylboronic acid $(300 \mu \mathrm{g})$ with either cefotaxime $(30 \mu \mathrm{g})$ or ceftazidime $(30 \mu \mathrm{g})$ (Yagi et al., 2005), aminophenylboronic acid hemisulfate (APBA) $(300 \mu \mathrm{g})$ with either cefotetan $(30 \mu \mathrm{g})$ or ceftazidime $(30 \mu \mathrm{g})$ (Tenover et al., 2009), 2-benzothienylboronic acid $(64 \mu \mathrm{g})$ with cefpodoxime $(10 \mu \mathrm{g})$ both with and without $1 \mu \mathrm{g}$ of clavulanic acid (Brenwald et al., 2005), cloxacillin $(100 \mu \mathrm{g})$ with cefoxitin $(30 \mu \mathrm{g})$ (Brenwald et al., 2005), and cloxacillin $(200 \mu \mathrm{g})$ with cefoxitin $(30 \mu \mathrm{g})$ (Tan et al., 2009). Blank discs with inhibitor alone were used to detect intrinsic antimicrobial activity of the inhibitor.

MAST ID D68C disc test. A 0.5 McFarland suspension of the test isolate was inoculated evenly on $\mathrm{MH}$ agar, then one each of four discs (A-D) were placed onto the agar in accordance with the manufacturer's instructions (Mast Group). Disc A contained cefpodoxime $(10 \mu \mathrm{g})$, disc B contained cefpodoxime $(10 \mu \mathrm{g})$ and an unidentified ESBL inhibitor, disc C contained cefpodoxime $(10 \mu \mathrm{g})$ and an unidentified AmpC inhibitor, and disc D contained cefpodoxime $(10 \mu \mathrm{g})$ and both the AmpC and ESBL inhibitors. Incubation was for $18-24 \mathrm{~h}$ in room air at $35{ }^{\circ} \mathrm{C}$. A zone difference of $\geqslant 5 \mathrm{~mm}$ between disc $\mathrm{C}$ and disc $\mathrm{A}$, or between disc $\mathrm{D}$ and disc $\mathrm{B}$, was taken to indicate the isolated presence of a $\mathrm{Bla}_{\mathrm{AmpC}}$ (Fig. 1b), whereas a zone difference of $\geqslant 5 \mathrm{~mm}$ between disc $\mathrm{D}$ and disc $\mathrm{C}$, but $<5 \mathrm{~mm}$ difference between disc $\mathrm{B}$ and disc $\mathrm{A}$, was taken to indicate the presence of both a $\mathrm{Bla}_{\mathrm{AmpC}}$ and an ESBL.

AmpC Etest. Double-sided Etest strips (BioMérieux) containing cefotetan $\left(0.5-32 \mu \mathrm{g} \mathrm{ml}^{-1}\right)$ with and without cloxacillin were applied to $\mathrm{MH}$ agar that had been inoculated evenly with a 0.5 McFarland suspension of the test isolate in accordance with the manufacturer's 


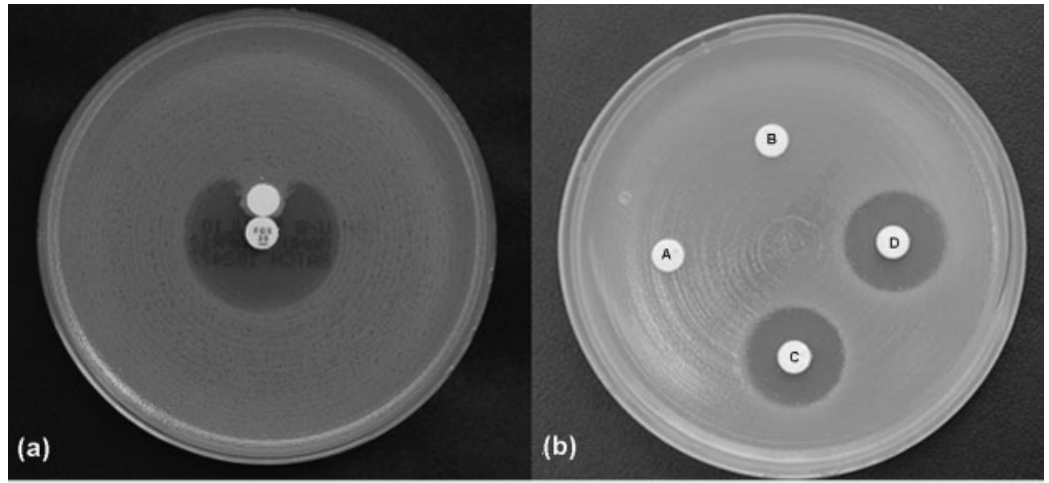

Fig. 1. (a) A positive result obtained from $B a_{A m p C}$ confirmatory testing using the TrisEDTA test. This shows a zone of inhibition of $E$. coli ATCC 25922 surrounding a cefoxitin disc, with indentation adjacent to a Tris-EDTAimpregnated disc smeared with several colonies of the test isolate. (b) A positive result obtained from $B \mathrm{a}_{\mathrm{AmpC}}$ confirmatory testing using the MAST ID D68C disc test. This shows a difference in inhibitory zone size of $\geqslant 5 \mathrm{~mm}$ between discs containing cefpodoxime with (C, D) and without (A, B) an unidentified $\mathrm{Bla}_{\mathrm{AmpC}} \mathrm{L}$ inhibitor.

instructions. After overnight incubation, an eightfold reduction in cefotetan MIC in the presence of cloxacillin, the presence of a 'phantom' zone of inhibition or deformation of the inhibition ellipse was interpreted as presence of a $\mathrm{Bla}_{\mathrm{AmpC}}$. The result for isolates with a cefotetan MIC $>32 \mu \mathrm{g} \mathrm{ml}^{-1}$ both with and without cloxacillin were considered indeterminate.

Cica-Beta chromogenic test. This test, which utilizes a chromogenic oxyiminocephalosporin substrate (HMRZ-86), was performed as described by Livermore et al. (2007).

Three-dimensional enzyme extraction assay. This assay was performed as described by Coudron et al. (2000). In view of the fact that carbapenemases may hydrolyse cefoxitin, the presence of enhanced growth of organisms at the point on the agar surface where the slit intersected the zone of inhibition was only taken as evidence of $\mathrm{Bla}_{\mathrm{AmpC}}$ when in combination with negative carbapenemase testing.

PCR. Detection of plasmid-borne genes encoding $\mathrm{Bla}_{\mathrm{AmpC}}$ was performed based upon the method described by Pérez-Pérez \& Hanson (2002). This multiplex nucleic acid amplification assay utilizes PCR primers specific for $b l a_{\mathrm{DHA}}, b l a_{\mathrm{ACC}}, b l a_{\mathrm{MOX}}, b l a_{\mathrm{CIT}}$, $b l a_{\mathrm{FOX}}$ and $b l a_{\mathrm{EBC}}$ and is capable of detecting six phylogenetic families of plasmid-mediated $\mathrm{Bla}_{\mathrm{AmpC}}$ on the basis of amplicon size. All positive PCR results were confirmed by monoplex PCR using individual primer sets with a primer concentration of $0.2 \mu \mathrm{M}$. The identity of PCR products was established by DNA sequencing performed using the Big Dye terminator system and a 3130XL instrument (Applied Biosystems), followed by a GenBank BLASTN search.

Performance and interpretation of the phenotypic testing was performed without reference to the results of PCR assays. E. coli ATCC 25922 (wild-type) was used as a negative control for all tests. Genotypically well-characterized $\mathrm{Bla}_{\mathrm{AmpC}^{-}}$-xpressing K. pneumoniae served as positive controls for phenotypic testing. For PCR testing, genotypically confirmed representatives of each of six phylogenetic $\mathrm{Bla}_{\mathrm{AmpC}}$ families acted as positive controls. For this purpose, an E. coli expressing FOX $\beta$-lactamase (J53 pMG252) was kindly provided by George Jacoby (Lahey Clinic, Burlington, MA, USA). John Iredell (ICPMR, Westmead Hospital, Sydney, Australia) kindly provided $K$. pneumoniae expressing CMY-2 (Jie091) and DHA $\beta$-lactamases (Jie142), an Enterobacter sp. expressing the EBC $\beta$-lactamase (W27Agr06), an Aeromonas sp. expressing the MOX $\beta$-lactamase (Ahc09) and a Hafnia alvei expressing the ACC $\beta$-lactamase (Hav09).

For the purpose of analysis, for all isolates other than E. coli, the reference method was detection of plasmid-encoded Bla $\mathrm{AmpC}_{\mathrm{C}}$ using the PCR assay. For E. coli, two reference methods were used for
Bla $_{\mathrm{AmpC}}$ detection: a positive PCR assay was interpreted as evidence of a plasmid-encoded $\mathrm{Bla}_{\mathrm{AmpC}}$, whereas the combination of a negative PCR assay, a positive three-dimensional enzyme extraction assay and a negative carbapenemase test was taken to represent hyperproduction of chromosomally encoded $\mathrm{Bla}_{\mathrm{AmpC}}$. The three-dimensional enzyme extraction assay selected for this purpose had previously been shown to have $100 \%$ sensitivity and specificity for $\mathrm{Bla}_{\mathrm{AmpC}}$ detection (Yong et al., 2002).

Fisher's exact test was used to compare proportions using GraphPad Instat 3.06 .

\section{RESULTS}

Seventy-four $(30 \%)$ isolates demonstrated evidence of a Bla $_{A m p C}$ using the reference method, including $47 \mathrm{E}$. coli, $23 \mathrm{~K}$. pneumoniae, 3 P. mirabilis and 1 Salmonella sp. (Table 1). Of the 47 E. coli, 22 (47\%) had hyperproduction of chromosomal Bla $\mathrm{AmpC}_{\mathrm{m}}$ and $25(53 \%)$ had plasmidencoded $\mathrm{Bla}_{\mathrm{AmpC}}$. Of the study isolates with plasmidencoded $\mathrm{Bla}_{\mathrm{AmpC}}$, the proportion with CMY (77\%), DHA $(20 \%)$ and EBC (4\%) enzymes was in keeping with the known epidemiology of plasmid-encoded Bla $\mathrm{AmpC}_{\mathrm{m}}$ Australia (Ellem et al., 2009b). A previously recognized (Tan et al., 2009) association between certain bacterial species and $\mathrm{Bla}_{\mathrm{AmpC}}$ type was observed (i.e. K. pneumoniae and DHA-1, E. coli and CMY-2).

The test performance of various proposed screening and confirmatory tests for Bla $\mathrm{AmpC}_{\mathrm{C}}$ detection is shown in Tables 2 and 3 , respectively.

Comparison of the methods for detecting different Bla $\mathrm{AmpC}_{\mathrm{A}}$ families revealed that the inhibitor-based methods utilizing cloxacillin and cefoxitin were significantly less sensitive for detection of DHA enzymes compared to CMY enzymes ( $0 \%$ vs $58 \%$ for $100 \mu$ g cloxacillin, $P=0.0036$; $0 \%$ vs $78 \%$ for $200 \mu \mathrm{g}$ of cloxacillin, $P<0.0001)$.

Fig. 2(a, b) are histograms demonstrating the inhibitory zone sizes for cefoxitin and cefotetan as determined by disc diffusion, separated according to the presence or absence of Bla $_{A m p C}$. Using CLSI breakpoints, non-susceptibility to cefoxitin rather than cefotetan was a more sensitive $(97 \%$ vs $47 \%, P<0.0001)$ but less specific $(64 \%$ vs $95 \%$, $P<0.0001)$ means of screening for the presence of Bla $\mathrm{AmpC}_{\text {. }}$. 
Table 1. Bla $\mathrm{AmpC}$-expressing isolates, according to bacterial species and enzyme family

\begin{tabular}{|c|c|c|c|c|c|}
\hline \multirow[t]{2}{*}{ Species } & \multirow[t]{2}{*}{ No. of isolates } & \multicolumn{3}{|c|}{ Plasmid-encoded Bla $_{\text {AmpC }}$ family } & \multirow{2}{*}{$\begin{array}{l}\text { Hyperproduction of } \\
\text { chromosomal Bla } \\
\text { AmpC }\end{array}$} \\
\hline & & $\mathrm{CMY}^{\star}$ & DHA $\dagger$ & $\mathrm{EBC} \neq$ & \\
\hline E. coli & 47 & 25 & 0 & 0 & 22 \\
\hline K. pneumoniae & 23 & 11 & 8 & 2 & 0 \\
\hline P. mirabilis & 3 & 2 & 1 & 0 & 0 \\
\hline Salmonella sp. & 1 & 1 & 0 & 0 & 0 \\
\hline
\end{tabular}

${ }^{*}$ All were CMY-2, except a single CMY-14-like $\mathrm{Bla}_{\mathrm{AmpC}}$ in an E. coli isolate.

$\dagger$ All were DHA- 1 .

¥Unable to distinguish between ACT-1/MIR-1.

Five isolates co-expressed both $\mathrm{Bla}_{\mathrm{AmpC}}$ and ESBL activity. Compared to isolates that expressed only $\mathrm{Bla}_{\mathrm{AmpC}}$, the performance of detection methods for these five isolates did not differ significantly, with the exception of the inhibitor-based method utilizing cefpodoxime and 2benzothienylboronic acid, which was less sensitive for detection of co-producers of $\mathrm{Bla}_{\mathrm{AmpC}}$ and $\mathrm{ESBL}(0 \%$ vs $69 \%, P=0.0159)$.

AmpC Etest results for $10(4 \%)$ of the isolates were indeterminate. Of these, six expressed carbapenemases, one an ESBL and one a $\mathrm{Bla}_{\mathrm{AmpC}}$, whilst the remainder had no identifiable $\beta$-lactamases.

When tested in isolation, none of the $\mathrm{Bla}_{\mathrm{AmpC}}$ inhibitors displayed intrinsic antimicrobial activity against the study isolates.

\section{DISCUSSION}

A lack of consensus regarding optimal methods for $\mathrm{Bla}_{\mathrm{AmpC}}$ detection is a major barrier to defining their epidemiology and understanding their clinical significance (Sidjabat et al., 2009). A mechanism-based approach to $\mathrm{Bla}_{\mathrm{AmpC}}$ detection may reduce erroneous cephalosporin susceptibility reporting (Thomson, 2010), and enable laboratories to explain a significant proportion of falsepositive ESBL screening and false-negative ESBL confirmatory tests (Robberts et al., 2009). In the face of reducing development of new antibiotics, the early identification of, and application of basic infection control principles to, patients harbouring bacteria with broad-spectrum resistance mechanisms becomes paramount (Thomson, 2010). For these reasons, the development of standardized guidelines for detection of broad-spectrum $\beta$-lactamases was recently identified as a priority for both national and international laboratory method committees (Giske et al., 2009).

The large number of isolates tested, the breadth of $\mathrm{Bla}_{\mathrm{AmpC}}$ detection methods assessed and the inclusion of isolates possessing ESBL and carbapenemases enabled a comprehensive comparison of test performance in our study. Akin

Table 2. Test performance of proposed $B a_{A m p C}$ screening methods

\begin{tabular}{|c|c|c|c|}
\hline Criterion & Method & Sensitivity & Specificity \\
\hline Cefoxitin non-susceptibility ${ }^{*}$ & $\mathrm{MIC} \dagger$ & $70 / 71(99 \%)$ & $73 / 164(45 \%)$ \\
\hline Cefoxitin non-susceptibility & Disc diffusion & $72 / 74(97 \%)$ & $110 / 172(64 \%)$ \\
\hline Cefotetan non-susceptibility & Disc diffusion & $35 / 74(47 \%)$ & $163 / 172(95 \%)$ \\
\hline $\begin{array}{l}\text { Cefoxitin non-susceptibility }{ }^{*}+ \\
\text { ceftazidime non-susceptibility }\end{array}$ & Disc diffusion & $53 / 74(72 \%)$ & $159 / 172(92 \%)$ \\
\hline $\begin{array}{l}\text { Positive ESBL screen }{ }^{\star}+ \\
\text { negative ESBL confirmatory test }{ }^{*}\end{array}$ & $\begin{array}{l}\text { Disc diffusion and/or MIC } \\
\text { Disc potentiation }\end{array}$ & $66 / 74(89 \%)$ & $143 / 172(83 \%)$ \\
\hline $\begin{array}{l}\text { Positive ESBL screen }{ }^{\star}+ \\
\text { cefoxitin non-susceptibility }{ }^{\star}\end{array}$ & $\begin{array}{l}\text { Disc diffusion and/or MIC } \\
\text { Disc diffusion }\end{array}$ & $70 / 74(95 \%)$ & $144 / 172(84 \%)$ \\
\hline $\begin{array}{l}\text { Positive ESBL screen }{ }^{\star}+ \\
\text { cefepime susceptibility }\end{array}$ & $\begin{array}{l}\text { Disc diffusion and/or MIC } \\
\text { MIC } \dagger\end{array}$ & $68 / 71(96 \%)$ & $150 / 164(91 \%)$ \\
\hline
\end{tabular}

${ }^{\star}$ As per CLSI criteria (CLSI, 2010).

$\dagger$ Excluding the 11 P.mirabilis isolates. 
Table 3. Test performance of proposed $B \mathrm{a}_{\mathrm{AmpC}}$ confirmatory methods

\begin{tabular}{|lcc|}
\hline Method & Sensitivity & Specificity \\
\hline Modified (cefoxitin) Hodge test & $54 / 74(73 \%)$ & $163 / 172(95 \%)$ \\
Tris-EDTA test & $72 / 74(97 \%)$ & $169 / 172(98 \%)$ \\
Saline disc test & $64 / 74(86 \%)$ & $162 / 172(94 \%)$ \\
Phenylboronic acid $(400 \mu \mathrm{g})^{*}$ & & \\
$\quad$ Cefoxitin & $49 / 74(66 \%)$ & $168 / 172(98 \%)$ \\
Cefotetan & $57 / 74(77 \%)$ & $170 / 172(99 \%)$ \\
Phenylboronic acid $(300 \mu \mathrm{g})^{*}$ & & \\
Cefotaxime & $33 / 74(45 \%)$ & $171 / 172(99 \%)$ \\
Ceftazidime & $58 / 74(78 \%)$ & $171 / 172(99 \%)$ \\
APBA $(300 \mu \mathrm{g})^{*}$ & & \\
Cefotetan & $51 / 74(69 \%)$ & $171 / 172(99 \%)$ \\
Ceftazidime & $58 / 74(78 \%)$ & $171 / 172(99 \%)$ \\
2-Benzothienylboronic acid $(64 \mu \mathrm{g})^{*}$ & & \\
Cefpodoxime & $45 / 74(61 \%)$ & $172 / 172(100 \%)$ \\
Cefpodoxime + clavulanic acid & $45 / 74(61 \%)$ & $151 / 172(88 \%)$ \\
Cloxacillin $(100 \mu \mathrm{g})^{*}$ & & \\
Cefoxitin & $39 / 74(53 \%)$ & $171 / 172(99 \%)$ \\
Cloxacillin $(200 \mu \mathrm{g})^{\star}$ & & \\
Cefoxitin & $52 / 74(70 \%)$ & $172 / 172(100 \%)$ \\
MAST ID D68C disc test & $71 / 74(96 \%)$ & $169 / 172(98 \%)$ \\
AmpC Etest $\dagger$ & $62 / 74(84 \%)$ & $161 / 172(94 \%)$ \\
Cica-Beta test & $14 / 74(19 \%)$ & $172 / 172(100 \%)$ \\
\hline
\end{tabular}

${ }^{\star}$ Increase in inhibitory zone of $\geqslant 3 \mathrm{~mm}$ was the criterion for a positive result.

$\dagger 10$ indeterminate results were included in the analysis. to ESBL detection, the low prevalence of $\mathrm{Bla}_{\mathrm{AmpC}}$ favours a two-step process in which a highly sensitive screening test is followed by a more specific confirmatory test (Doi \& Paterson, 2007). Interpretative cut-off points used in this study were selected to support a two-step evaluative test process.

Cephamycin hydrolysis distinguishes $\mathrm{Bla}_{\mathrm{AmpC}}$ from many other $\beta$-lactamases. We found that non-susceptibility to cefoxitin, rather than cefotetan, was a more useful screening tool for $\mathrm{Bla}_{\mathrm{AmpC}}$ detection, with a sensitivity of $\geqslant 97 \%$ using either an MIC or disc-based method. The use of a cefoxitin inhibitory zone size less than the CLSI breakpoint (or a higher MIC) improved specificity with marginal loss of sensitivity (Fig. 2a, Table 2), as has been shown elsewhere (Coudron et al., 2000). Other mechanisms of cephamycin resistance (e.g. porin deficiency, carbapenemases) and the fact that the cefoxitin CLSI breakpoint is only twice the modal wild-type cefoxitin MIC (Livermore, 1995) weakens the specificity of a screening approach using cefoxitin non-susceptibility. A positive ESBL screen is an adequate alternative (sensitivity $96 \%$ ) that is familiar to most laboratories. The use of combined screening tests improved specificity, but with variable impact on sensitivity. The optimal screening test combination was a positive ESBL screen plus cefepime susceptibility, which had a sensitivity of $96 \%$ and specificity of $91 \%$.

The performance of confirmatory tests for $\mathrm{Bla}_{\mathrm{AmpC}}$ detection varied widely, ranging in sensitivity from $19 \%$ to $97 \%$ and in specificity from $88 \%$ to $100 \%$. Only two
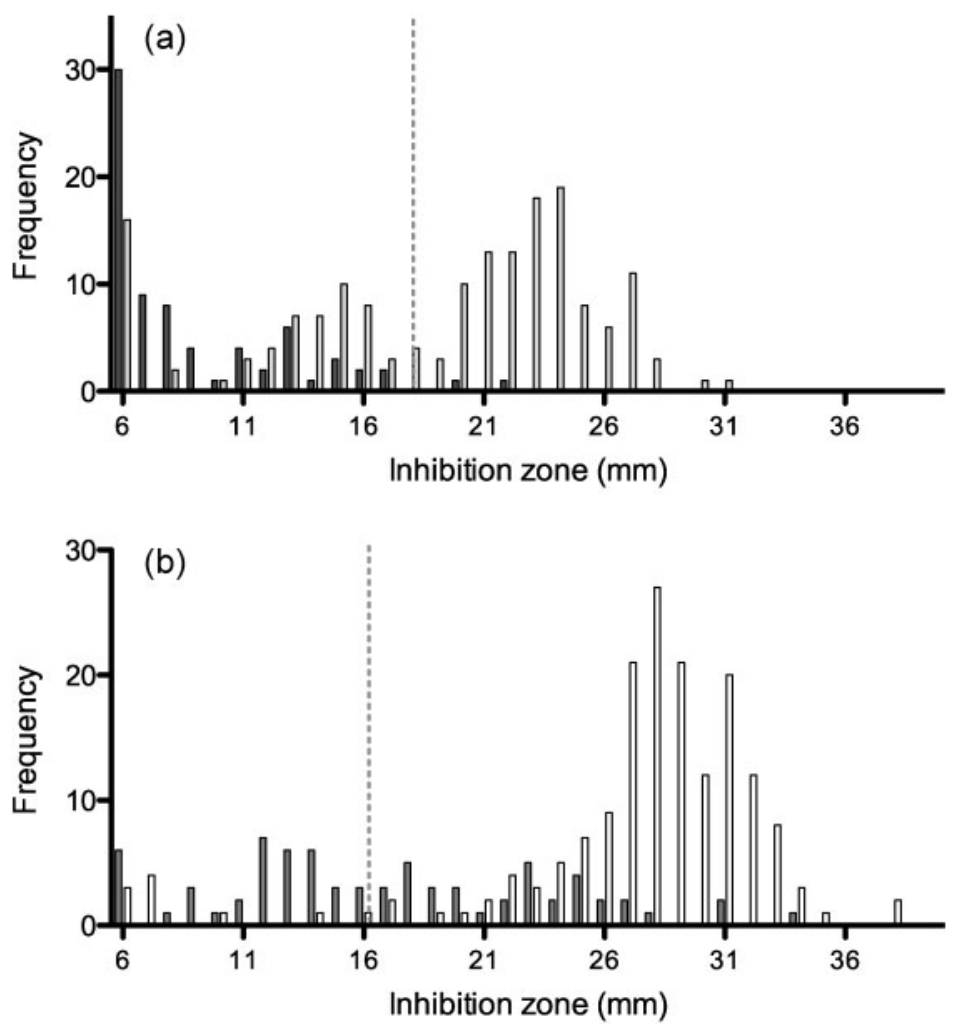

Fig. 2. (a) Cefoxitin inhibitory zone sizes for $\mathrm{Bla}_{\mathrm{AmpC}}$-containing (dark grey bars) and non$\mathrm{Bla}_{\mathrm{AmpC}}$-containing (light grey bars) isolates. The CLSI breakpoint for non-susceptibility is $<18 \mathrm{~mm}$ (dotted line) (CLSI, 2010). (b) Cefotetan inhibitory zone sizes for $\mathrm{Bla}_{\mathrm{AmpC}^{-}}$ containing (dark grey bars) and non-Bla $\mathrm{AmpC}^{-}$ containing (white bars) isolates. The CLSI breakpoint for non-susceptibility is $<16 \mathrm{~mm}$ (dotted line) (CLSI, 2010). 
methods, the Tris-EDTA and MAST ID D68C disc test, had both sensitivity and specificity $>90 \%$. The Tris-EDTA test performed better than the other biological assays, presumably because Tris-EDTA improves the release of $\beta$-lactamases by permeabilizing Gram-negative cells (Thomson, 2010). Another property of Tris-EDTA, the inhibition of MBL carbapenemase activity (Thomson, 2010), improved the specificity of this assay by preventing cefoxitin hydrolysis by this enzyme, as was demonstrated in six of the seven MBLexpressing isolates. The high sensitivity and specificity of this method has been reported in other studies (Black et al., 2005a). Whilst the commercial MAST ID D68C disc test performed as well as the in-house Tris-EDTA method, lower acquisition costs may make the latter more cost effective. The AmpC Etest was easy to use, but was unsuitable for testing for $\mathrm{Bla}_{\mathrm{AmpC}}$ in $4 \%$ of isolates, although the majority of those did not possess an $\mathrm{Bla}_{\mathrm{AmpC}}$. Test performance could be improved by developing an Etest strip with a broader MIC range. The poor performance of the chromogenic Cica-Beta test is consistent with findings of other studies (Montgomery et al., 2008).

Inhibitor-based assays are the most widely published $\mathrm{Bla}_{\mathrm{AmpC}}$ confirmatory method. We found the sensitivity and specificity of all the inhibitor-based methods to be lower than previously described (Tan et al., 2008). In contrast to other studies suggesting that cefotaxime was the optimal substrate (Hope et al., 2008), we found ceftazidime to be superior, perhaps explained by preferential hydrolysis of this cephalosporin by $\mathrm{Bla}_{\mathrm{AmpC}}$ (Philippon et al., 2002). When used at lower concentrations, cloxacillin appeared to have inferior sensitivity compared to the boronic acid compounds. However, comparisons must be made with caution as the concentration of each inhibitor that optimizes test performance remains to be defined. When used with the optimal cephalosporin substrate, phenylboronic acid and ABPA were superior to 2-benzothienylboronic acid, but still lacked sensitivity. Notably, all the boronic-acid-based assays produced false-positive results when tested on the KPC carbapenemase-producing isolate. Boronic acid is a known inhibitor of this enzyme (Thomson, 2010). Establishing the optimal inhibitor type, inhibitor concentration, cephalosporin substrate(s) and interpretative cut-offs for this group of tests requires further research.

Variation in test performance between $\mathrm{Bla}_{\mathrm{AmpC}}$ families is known to occur. For example, due to differing substrate specificity, cephamycin non-susceptibility is a less sensitive screening tool for the ACC family of enzymes (Tan et al., 2009). We observed differing sensitivity of cloxacillin inhibitor-based assays for detection of CMY and DHA enzymes, which may be explained by variation in the inhibitory capacity of cloxacillin between $\mathrm{Bla}_{\mathrm{AmpC}}$ families (Philippon et al., 2002). These unique findings warrant further investigation on a broader range of bacterial species and $\mathrm{Bla}_{\mathrm{AmpC}}$ families.

Our study has several limitations. We included relatively few isolates with co-production of both $\mathrm{Bla}_{\mathrm{AmpC}}$ and ESBL.
This infrequently described combination is known to weaken the performance of inhibitor-based methods of $\mathrm{Bla}_{\mathrm{AmpC}}$ detection (Tenover et al., 2009), as was observed for the cefpodoxime and 2-benzothienylboronic acid combination in our study. To address this, further investigation of methods simultaneously utilizing clavulanic acid and $\mathrm{Bla}_{\mathrm{AmpC}}$ inhibitors is warranted. Secondly, the geographical distribution of plasmid-encoded $\mathrm{Bla}_{\mathrm{AmpC}}$ families varies widely (Alvarez et al., 2004) and as we demonstrated, the performance of phenotypic tests may differ between these families, potentially restricting the applicability of our findings to other settings. However, in our study the majority of plasmid-encoded $\mathrm{Bla}_{\mathrm{AmpC}}$ were of the CMY type, the most prevalent type in all other regions of the world (Jacoby, 2009). Finally, it is possible that other $\mathrm{Bla}_{\mathrm{AmpC}}$ families, not detected by the multiplex PCR assay used, were present in our study. A novel plasmid-encoded $\mathrm{Bla}_{\mathrm{AmpC}}, \mathrm{CFE}-1$, has recently been described, although its prevalence remains to be defined (Nakano et al., 2004).

In conclusion, a number of $\mathrm{Bla}_{\mathrm{AmpC}}$ screening options exist. These have excellent sensitivity but the performance of confirmatory tests differs widely. Further investigation of optimal substrates, inhibitory type, inhibitor concentration and interpretative cut-offs is needed in order to improve the performance of the inhibitor-based assays. A simple disc-based protocol utilizing cefoxitin nonsusceptibility as a screening method, then using the TrisEDTA method for confirmation, could conveniently be incorporated into laboratory workflow and would detect Bla $_{\mathrm{AmpC}}$ with a sensitivity of $95 \%$ and a specificity of $98 \%$.

\section{ACKNOWLEDGEMENTS}

This work was supported in part by a PathWest QEII Medical and Scientific Research Grant. We would like to thank Sue-Ellen Blackaby (Blackaby Diagnostics, Australia), who provided the Cica-Beta chromogenic test kits, and bioMérieux (France) for supplying the AmpC Etests. We appreciate the assistance of our colleagues in the Department of Microbiology, PathWest Laboratory Medicine, WA, with collection of clinical isolates. We thank George Jacoby (Lahey Clinic, Burlington, MA) for kindly reviewing the manuscript prior to submission.

\section{REFERENCES}

Alvarez, M., Tran, J. H., Chow, N. \& Jacoby, G. A. (2004). Epidemiology of conjugative plasmid-mediated AmpC beta-lactamases in the United States. Antimicrob Agents Chemother 48, 533-537.

Black, J. A., Moland, E. S. \& Thomson, K. S. (2005a). AmpC disk test for detection of plasmid-mediated AmpC beta-lactamases in Enterobacteriaceae lacking chromosomal AmpC beta-lactamases. J Clin Microbiol 43, 3110-3113.

Black, J. A., Thomson, K. S., Buynak, J. D. \& Pitout, J. D. (2005b). Evaluation of beta-lactamase inhibitors in disk tests for detection of plasmid-mediated AmpC beta-lactamases in well-characterized clinical strains of Klebsiella spp. J Clin Microbiol 43, 4168-4171.

Brenwald, N. P., Jevons, G., Andrews, J., Ang, L. \& Fraise, A. P. (2005). Disc methods for detecting AmpC $\beta$-lactamase-producing 
clinical isolates of Escherichia coli and Klebsiella pneumoniae. J Antimicrob Chemother 56, 600-601.

CLSI (2009). Methods for Dilution Antimicrobial Susceptibility Tests for Bacteria that Grow Aerobically, M7-A8. Wayne, PA: Clinical Laboratory Standards Institute.

CLSI (2010). Performance Standards for Antimicrobial Susceptibility Testing, M100-S20. Wayne, PA: Clinical and Laboratory Standards Institute.

Coudron, P. E. (2005). Inhibitor-based methods for detection of plasmid-mediated AmpC beta-lactamases in Klebsiella spp., Escherichia coli, and Proteus mirabilis. J Clin Microbiol 43, 4163-4167.

Coudron, P. E., Moland, E. S. \& Thomson, K. S. (2000). Occurrence and detection of AmpC $\beta$-lactamases among Escherichia coli, Klebsiella pneumoniae, and Proteus mirabilis isolates at a veterans medical center. J Clin Microbiol 38, 1791-1796.

Ding, H., Yang, Y., Lu, Q., Wang, Y., Chen, Y., Deng, L., Wang, A., Deng, Q., Zhang, H. \& other authors (2008). The prevalence of plasmid-mediated AmpC beta-lactamases among clinical isolates of Escherichia coli and Klebsiella pneumoniae from five children's hospitals in China. Eur J Clin Microbiol Infect Dis 27, 915-921.

Doi, Y. \& Paterson, D. L. (2007). Detection of plasmid-mediated class C beta-lactamases. Int J Infect Dis 11, 191-197.

Ellem, J., Thomas, L., OIma, T. \& Iredell, J. (2009a). Comparison and evaluation of a newly developed Mast 4-Disc test for the detection of plasmid-mediated AmpC beta-lactamases. In Australian Society for Antimicrobials Conference, Sydney, Australia. Abstract no. P2.3.

Ellem, J., Thomas, L., Olma, T. \& Iredell, J. (2009b). Rapid detection of AmpC beta-lactamases by multiplex real-time PCR and melt curve analysis. In Australian Society for Antimicrobials Conference, Sydney, Australia. Abstract no. P2.4.

Giske, C. G., Sundsfjord, A. S., Kahlmeter, G., Woodford, N., Nordmann, P., Paterson, D. L., Cantón, R. \& Walsh, T. R. (2009). Redefining extended-spectrum beta-lactamases: balancing science and clinical need. J Antimicrob Chemother 63, 1-4.

Hope, R., Warner, M. \& Livermore, D. M. (2008). Phenotypic AmpC detection - which inhibitor is best? In 18th European Congresses of Clinical Microbiology and Infectious Diseases Conference, Barcelona, Spain. Abstract no. P876.

Jacoby, G. A. (2009). AmpC beta-lactamases. Clin Microbiol Rev 22, 161-182.

Kohner, P. C., Robberts, F. J., Cockerill, F. R., III \& Patel, R. (2009), Cephalosporin MIC distribution of extended-spectrum-betalactamase- and pAmpC-producing Escherichia coli and Klebsiella species. J Clin Microbiol 47, 2419-2425.

Livermore, D. M. (1995). $\beta$-Lactamases in laboratory and clinical resistance. Clin Microbiol Rev 8, 557-584.

Livermore, D. M., Warner, M. \& Mushtaq, S. (2007). Evaluation of the chromogenic Cica- $\beta$-Test for detecting extended-spectrum, AmpC and metallo- $\beta$-lactamases. J Antimicrob Chemother 60, 1375-1379.

Moland, E. S., Black, J. A., Ourada, J., Reisbig, M. D., Hanson, N. D. \& Thomson, K. S. (2002). Occurrence of newer beta-lactamases in Klebsiella pneumoniae isolates from 24 U.S. hospitals. Antimicrob Agents Chemother 46, 3837-3842.

Montgomery, J., Nakos, J. \& Gurtler, V. (2008). Comparison of phenotypic tests for detection of plasmid-mediated AmpC betalactamases. In Australian Society for Antimicrobials Conference, Sydney, Australia. Abstract no. P2.2.
Mulvey, M. R., Bryce, E., Boyd, D. A., Ofner-Agostini, M., Land, A. M., Simor, A. E. \& Paton, S. (2005). Molecular characterization of cefoxitin-resistant Escherichia coli from Canadian hospitals. Antimicrob Agents Chemother 49, 358-365.

Munier, G. K., Johnson, C. L., Snyder, J. W., Moland, E. S., Hanson, N. D. \& Thomson, K. S. (2010). Positive extended-spectrum-betalactamase (ESBL) screening results may be due to AmpC betalactamases more often than to ESBLs. J Clin Microbiol 48, 673-674.

Nakano, R., Okamoto, R., Nakano, Y., Kaneko, K., Okitsu, N., Hosaka, Y. \& Inoue, M. (2004). CFE-1, a novel plasmid-encoded AmpC $\beta$-lactamase with an $a m p R$ gene originating from Citrobacter freundii. Antimicrob Agents Chemother 48, 1151-1158.

Pérez-Pérez, F. J. \& Hanson, N. D. (2002). Detection of plasmidmediated AmpC beta-lactamase genes in clinical isolates by using multiplex PCR. J Clin Microbiol 40, 2153-2162.

Philippon, A., Arlet, G. \& Jacoby, G. A. (2002). Plasmid-determined AmpC-type beta-lactamases. Antimicrob Agents Chemother 46, 1-11.

Robberts, F. J. L., Kohner, P. C. \& Patel, R. (2009). Unreliable extended-spectrum $\beta$-lactamase detection in the presence of plasmidmediated AmpC in Escherichia coli clinical isolates. J Clin Microbiol 47, 358-361.

Senda, K., Arakawa, Y., Ichiyama, S., Nakashima, K., Ito, H., Ohsuka, S., Shimokata, K., Kato, N. \& Ohta, M. (1996). PCR detection of metallo- $\beta$-lactamase gene $\left(b l a_{\mathrm{IMP}}\right)$ in gram-negative rods resistant to broad-spectrum $\beta$-lactams. J Clin Microbiol 34, 29092913.

Sidjabat, H. E., Paterson, D. L., Qureshi, Z. A., Adams-Haduch, J. M., O’Keefe, A., Pascual, A., Rodríguez-Baño, J. \& Doi, Y. (2009). Clinical features and molecular epidemiology of CMY-type betalactamase-producing Escherichia coli. Clin Infect Dis 48, 739-744.

Singhal, S., Mathur, T., Khan, S., Upadhyay, D. J., Chugh, S., Gaind, R. \& Rattan, A. (2005). Evaluation of methods for AmpC betalactamase in gram negative clinical isolates from tertiary care hospitals. Indian J Med Microbiol 23, 120-124.

Tan, T. Y., Ng, S. Y., Teo, L., Koh, Y. \& Teok, C. H. (2008). Detection of plasmid-mediated AmpC in Escherichia coli, Klebsiella pneumoniae and Proteus mirabilis. J Clin Pathol 61, 642-644.

Tan, T. Y., Ng, L. S., He, J., Koh, T. H. \& Hsu, L. Y. (2009). Evaluation of screening methods to detect plasmid-mediated AmpC in Escherichia coli, Klebsiella pneumoniae, and Proteus mirabilis. Antimicrob Agents Chemother 53, 146-149.

Tenover, F. C., Emery, S. L., Spiegel, C. A., Bradford, P. A., Eells, S., Endimiani, A., Bonomo, R. A. \& McGowan, J. E., Jr (2009). Identification of plasmid-mediated AmpC beta-lactamases in Escherichia coli, Klebsiella spp., and Proteus species can potentially improve reporting of cephalosporin susceptibility testing results. J Clin Microbiol 47, 294-299.

Thomson, K. S. (2010). Extended-spectrum-beta-lactamase, AmpC, and carbapenemase issues. J Clin Microbiol 48, 1019-1025.

Yagi, T., Wachino, J., Kurokawa, H., Suzuki, S., Yamane, K., Doi, Y., Shibata, N., Kato, H., Shibayama, K. \& Arakawa, Y. (2005). Practical methods using boronic acid compounds for identification of class $\mathrm{C}$ beta-lactamase-producing Klebsiella pneumoniae and Escherichia coli. J Clin Microbiol 43, 2551-2558.

Yong, D., Park, R., Yum, J. H., Lee, K., Choi, E. C. \& Chong, Y. (2002). Further modification of the Hodge test to screen AmpC betalactamase (CMY-1)-producing strains of Escherichia coli and Klebsiella pneumoniae. J Microbiol Methods 51, 407-410. 